\title{
ESTUDO DA APLICAÇÃO DE FERRAMENTAS DA QUALIDADE EM UMA LINHA DE ENVASE NUMA INDÚSTRIA ALIMENTÍCIA
}

\section{STUDY ABOUT THE IMPLEMENTATION OF QUALITY TOOLS ON A BOTTLING LINE IN FOOD INDUSTRY}

Juliana Matsumoto da Silva, Tamires Pereira, Andréia de Menezes Olivo

Universidade do Oeste Paulista - UNOESTE, Faculdade de Engenharias e Arquitetura e Urbanismo, Presidente Prudente, SP.

E-mail:juhmatsumoto@gmail.com.

RESUMO - Integrar conceitos de gestão da qualidade a melhoria contínua de processos é o grande desafio que se encontra para alcançar um programa contínuo de gestão. Cada vez mais as empresas buscam por ferramentas e técnicas de suporte gerencial para o alcance de diferenciais competitivos. O objetivo desse trabalho foi aplicar em uma indústria específica de alimentos e bebidas, ferramentas da qualidade para identificar e eliminar as causas que diminuem a eficiência da Tampadeira da linha de envase, equipamento responsável por tapar os frascos ao fim do envase. A metodologia utilizada foi uma pesquisa exploratória para coleta de dados na empresa mencionada, bibliografias descritivas e analíticas, baseadas em livros, artigos científicos, teses e normas regulamentadoras.

Palavras-chave: Gestão; Ferramentas; Controle; Qualidade; Melhoria Contínua.

ABSTRACT - Integrate quality management concepts to continuous process improvement is the big challenge is to achieve an ongoing management program. More and more companies look for tools and management support techniques to achieve competitive advantages. The aim of this study was to apply in a specific food and beverage industry, quality tools to identify and eliminate the causes that decrease the efficiency of Tampadeira the filling line equipment responsible for covering the bottles at the end of filling. The methodology used was an exploratory research to collect data on the mentioned company, descriptive and analytical bibliography based on books, scientific articles, theses and regulatory standards.

Keywords: Management; Tools; Control; Quality; Continuous

Recebido em: 09/08/2016 Improvement.

Revisado em: 25/08/2016

Aprovado em: 29/08/2016 


\section{INTRODUÇÃO}

Diante os avanços tecnológicos e o aumento na competitividade, toda organização passou a se preocupar com a qualidade do seu processo produtivo, pois a qualidade do produto final se tornou um diferencial e um pré-requisito a fim de diminuir as perdas, diminuir os desperdícios, diminuir os custos, aumentar a produtividade e aumentar os lucros em todo tipo de setor econômico, seja ela prestadora de serviços ou produtora de bens.

No transcorrer da evolução, as empresas desenvolveram métodos para atender as expectativas dos consumidores, se manterem no mercado competitivo, adquirindo consumidores mais satisfeitos e consequentemente mais exigentes (SELEME; HUMBERTO, 2012).

Desse modo, à medida que modernizavam e ampliavam os mercados, passaram a utilizar importantes ferramentas que auxiliavam o entendimento do processo produtivo e no desenvolvimento das metodologias usadas para identificação das principais falhas (SELEME; HUMBERTO, 2012).

Neste contexto, escolhemos uma linha de envase que não atende as expectativas da empresa, e o objetivo desse artigo é mostrar a efetiva utilização das ferramentas da qualidade para identificação dos maiores problemas, que consequentemente influenciam na eficiência diária e mensal.

\section{METODOLOGIA}

A linha estudada, comprada em 2014, é um equipamento destinado á fazer o envase de 51 tipos de produtos, com 3 operadores por turno, com 8 conjuntos de ferramentais específicos para cada tipo de embalagem, vários tipos de frascos, e vários tipos de tampas, onde as velocidades nominais também variam de produto para produto. A máquina é dividida em partes e o posicionador e a rotuladeira foram comprados com o intuito de atenderem com flexibilidade a grande variedade de formatos e tamanhos de frascos, reduzindo a mão de obra, organizando e posicionando corretamente com eficácia e rapidez, porém a mesma não funciona como esperado.

Durante a coleta de dados, para a aplicação das ferramentas, sendo elas Estratificação dos dados, Diagrama de Pareto, Diagrama de Ishikawa, Diagramas de Dispersão, e Carta Controle, foram realizados acompanhamentos diários, anotações de dados, esclarecimento de dúvidas com os operadores de linha e com supervisores da produção, de modo que facilitou a nossa compreensão sobre o caso, e nos mostrou as causas referentes à parada mais frequente, 
para então aplicar um plano de ação em busca de melhorias.

A empresa já possuía uma ficha de verificação para controle de paradas, onde cada linha recebe uma ficha no inicio do respectivo turno. Além da ficha de verificação a empresa conta com uma planilha, para apontamento diário dos dados, onde são anotadas informações necessárias para compreender o tempo de parada das máquinas. Através dessa planilha, selecionamos as paradas ocasionadas em Abril de 2016.

\section{RESULTADOS E DISCUSSÕES}

Segue a estratificação dos dados levantados (TABELA 1), onde é possível observar as paradas mais frequentes da linha em estudo, a tabela contém os minutos e as horas totais de tempo de parada durante o mês de Abril de 2016.

Tabela 01. Estratificação das paradas frequentes na linha de envase - Abril 2016

\begin{tabular}{|c|c|c|c|c|}
\hline \multicolumn{5}{|c|}{ PARADAS FREQUENTES NA LINHA DE ENVASE - ABRIL 2016} \\
\hline Balanço & Minutos & Horas & $\%$ & FC \% \\
\hline Parada na tampadeira & 580 & 9,67 & $32 \%$ & $32 \%$ \\
\hline Parada na rotuladeira & 511 & 8,52 & $28 \%$ & $60 \%$ \\
\hline Parada no posicionador de frascos & 228 & 3,80 & $13 \%$ & $73 \%$ \\
\hline Parada na enchedeira & 164 & 2,73 & $9 \%$ & $82 \%$ \\
\hline Falta de produto & 158 & 2,63 & $9 \%$ & $91 \%$ \\
\hline Encravamento de garrafas & 91 & 1,52 & $5 \%$ & $96 \%$ \\
\hline Problema na seladora de caixas & 72 & 1,20 & $4 \%$ & $100 \%$ \\
\hline
\end{tabular}

Fonte: (MATSUMOTO, 2016).

Uma vez feita à estratificação é possível visualizar de maneira clara, através do Gráfico de Pareto (FIGURA 1), 20\% das nossas causas principais que influenciam em $80 \%$ das consequências.

O Gráfico de Pareto é uma ferramenta desenvolvida através de estudos, no qual estabelece uma relação 20/80 (vinte para oitenta), ou seja, indica que a maior quantidade dos defeitos se refere a poucas causas (SELEME; HUMBERTO, 2012).

Nesse sentido, o gráfico nos permite identificar e classificar aqueles problemas de maior importância e que devem ser corrigidos primeiramente (SELEME; HUMBERTO, 2012). 


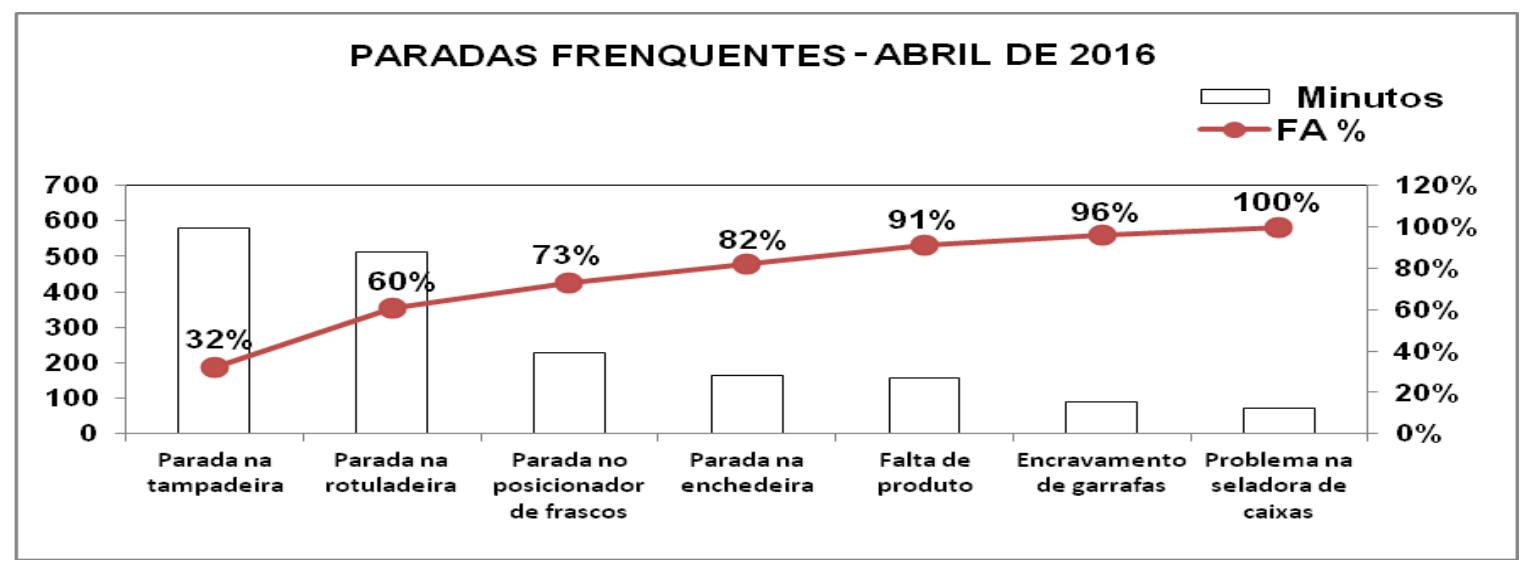

Figura 01. Gráfico de Pareto das paradas frequentes - Abril 2016

Fonte: (MATSUMOTO, 2016).

Ao visualizar o gráfico é possível observar que os $20 \%$ dos problemas responsáveis pelas paradas dessa linha ocorrem na Tampadeira, na Rotuladeira, no Posicionador de frascos e na Enchedeira, sendo estes os responsáveis por $80 \%$ das paradas ocasionadas.

A maior frequência se encontra na Tampadeira, onde as tampas são um dos principais motivos dessas paradas, pois, enroscam na esteira constantemente antes mesmo de serem fixadas nas garras responsáveis por tamparem os frascos, geralmente enroscam devido ao atrito da esteira sob a tampa, ou quando estão quebradas, e abertas.

As tampas orientais dificilmente quebram, porém, devido seu formato é a maior responsável por fazerem as tampas enroscarem, a segunda maior responsável são as tampas de Flip Top com lacres, muitas passam com a tampa aberta, ou com o lacre quebrado e acabam enroscando na esteira também. Outro grande problema no processo da Tampadeira é o sensor que falha com frequência e é responsável por ver se os frascos estão cheios, e aptos a serem tampados. Todos esses pequenos fatores fazem com que os frascos saiam da enchedeira sem tampa, atrapalhando todo o processo produtivo, e causando transtorno.

E devido à complexidade dos mesmos foi aplicado o Diagrama de Ishikawa apenas no motivo mais frequente, que como dito anteriormente se situa na Tampadeira, sendo $32 \%$ responsável pela maior frequência acumulada (FIGURA 2), com isso, dividimos os problemas através das possíveis causas que podem ser responsáveis pelas falhas críticas e foram baseadas nos $6 \mathrm{Ms}$ (medição, materiais, mão de obra, máquina, método e meio ambiente).

$$
\text { Com esse diagrama é possível }
$$
identificar as causas, e realizarmos análises verificando as ocorrências que apresentam ou não consequências, e nos 
possibilita a potencialização do primeiro e eliminação do segundo (SELEME; HUMBERTO, 2012).

É de fácil representação, fácil compreensão, e tem um formato de espinha de peixe como o próprio nome já indica, pois além de Diagrama de Ishikawa, também pode ser chamado de Espinha de Peixe ou Diagrama de Causa e Efeito (SELEME; HUMBERTO, 2012).
O mesmo também nos possibilita elaborar um plano de ação, que abordará as peculiaridades de cada uma, esse plano de ação pode ajudar a traçar objetivos, aumentar a responsabilidade das pessoas envolvidas no plano especifico e potencializar os resultados da empresa.

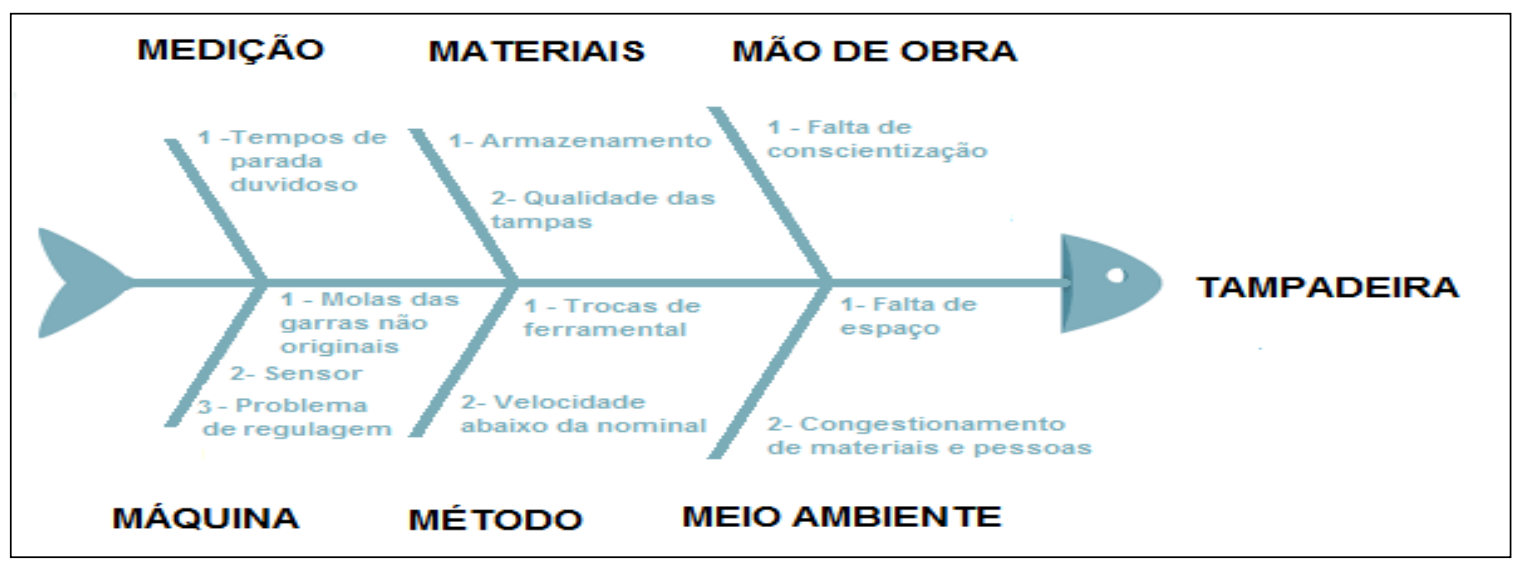

Figura 02. Diagrama de Ishikawa das causas de parada na Tampadeira.

Fonte: (MATSUMOTO, 2016).

Durante a identificação e sinalização das principais causas, foi possível apontar os maiores problemas como visto no diagrama, pois independente do tipo de produto que estava produzindo, a regulagem inadequada, os tempos de paradas que não são anotados criteriosamente, a forma de armazenamento, a falta de conscientização, a falta de treinamentos específicos, as trocas constantes de ferramentais, velocidade na maioria das vezes abaixo da nominal, falta de espaço, e congestionamento de materiais e pessoas, são fatores agravantes que acontecem frequentemente, e prejudicam o desempenho e o objetivo pelo qual a máquina foi comprada.

Na sequência, foi feito um Diagrama de Dispersão para analisar se há uma correlação entre a média de eficiência do frasco e seu fornecedor, e a quantidade de caixas produzidas. A Tabela 2 junto à Figura 3 mostram a exposição dos dados.

“O Diagrama de Dispersão permite identificar relações entre causas e efeitos 
pela interpretação gráfica das variáveis." ～(CUSTÓDIO, 2015, p. 28).

Tabela 02. Média de eficiência do frasco e seu fornecedor e a quantidade de caixas produzidas.

\begin{tabular}{|c|c|c|}
\hline FORNECEDOR E FRASCO & MÉDIA (\%) & QUANTIDADE \\
\hline OWENS (150 ML) & 28 & 3.475 \\
\hline GIACOMINI (500 ML) & 26 & 5.514 \\
\hline PLASTSAMER (750G, 780G, 820G) & 38 & 7.121 \\
\hline PREMIUM PLASTIC (150 ML) & 29 & 8.195 \\
\hline PLASTSAMER (170G, 185G, 190G) & 38 & 9.865 \\
\hline GIACOMINI (900 ML) & 45 & 11.309 \\
\hline PLASTSAMER (370G, 390G, 400G) & 44 & 15.776 \\
\hline
\end{tabular}

Fonte: (MATSUMOTO, 2016).

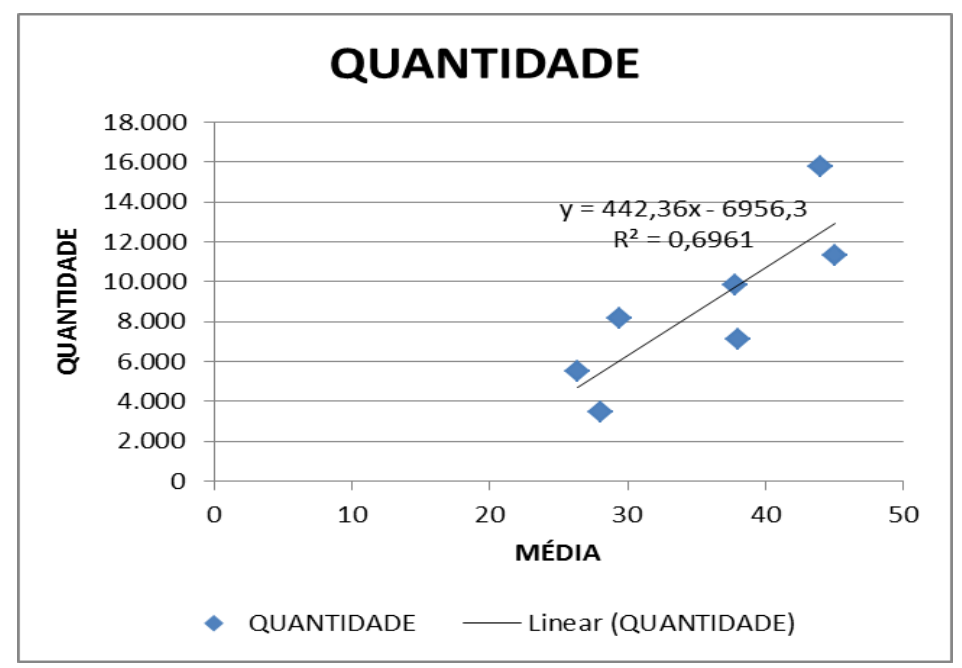

Figura 03. Gráfico de Dispersão - Correlação da média de eficiência do frasco e seu fornecedor, e a quantidade de caixas produzidas.

Fonte: (PEREIRA, 2016).

Pode-se afirmar que as variáveis se influenciam, embora a correlação seja baixa, ainda assim, o gráfico de dispersão e suas ferramentas mostra uma correlação de $69,61 \%$ entre as variáveis.

Já o gráfico de controle é um método que possibilita a análise da variação à qual um processo está submetido, e mostra se essa variação está dentro do intervalo médio esperado ou se há um desvio que deve ser investigado (BARROS; BONAFINI, 2014).

Uma análise estatística foi feita para monitorar o tempo de parada na Tampadeira, para isso utilizou-se o gráfico de controle, assim foi possível visualizar através da ferramenta momentos em que o tempo de parada estava fora do controle, além da faixa de variação que se encontra o tempo de 
parada na máquina. A Figura 4 a seguir ilustra a planilha utilizada. Os dados foram dispostos da seguinte forma: Tempo de parada de hora em hora, cada dia do mês em que houve paradas na máquina.

Figura 04. llustração da planilha para carta controle da Tampadeira.

\begin{tabular}{|c|c|c|c|c|c|c|c|c|c|}
\hline \multicolumn{10}{|c|}{ PARADAS NA TAMPADEIRA, PLANILHA PARA GRÁFICO DE CONTROLE } \\
\hline Data & Início & Término & Tempo & Data/Hora & |00:00 à 01:00 & 01:00 à 02:00 & 02:00 à 03:00 & |03:00 à $04: 00$ & $20: 00$ à $21: 00$ \\
\hline $02 / \mathrm{abr}$ & $09: 35$ & 09:55 & 20 & $02 / a b r$ & 0 & 0 & 0 & 0 & 0 \\
\hline $05 / \mathrm{abr}$ & $00: 20$ & $00: 35$ & 15 & $05 / \mathrm{abr}$ & 15 & 0 & 0 & 0 & 0 \\
\hline $05 / a b r$ & $16: 39$ & $16: 49$ & 10 & 08/abr & 30 & 0 & 0 & 0 & 0 \\
\hline 08/abr & $00: 30$ & 01:00 & 30 & 09/abr & 0 & 0 & 0 & 0 & 0 \\
\hline $09 / \mathrm{abr}$ & $07: 40$ & 08:00 & 20 & $11 / \mathrm{abr}$ & 30 & 0 & 0 & 0 & 15 \\
\hline $09 / \mathrm{abr}$ & 08:05 & $08: 20$ & 15 & $12 / \mathrm{abr}$ & 25 & 30 & 30 & 60 & 0 \\
\hline $11 / \mathrm{abr}$ & $00: 30$ & 01:00 & 30 & $13 / a b r$ & 0 & 0 & 0 & 0 & 0 \\
\hline $11 / \mathrm{abr}$ & $20: 00$ & $20: 15$ & 15 & $14 / \mathrm{abr}$ & 0 & 0 & 0 & 0 & 0 \\
\hline $12 / \mathrm{abr}$ & $00: 35$ & 01:30 & 55 & 19/abr & 0 & 0 & 0 & 0 & 0 \\
\hline $12 / \mathrm{abr}$ & $02: 30$ & 04:30 & 120 & $21 / a b r$ & 0 & 0 & 0 & 0 & 10 \\
\hline $12 / \mathrm{abr}$ & $15: 35$ & $15: 42$ & 7 & $22 / \mathrm{abr}$ & 0 & 0 & 0 & 0 & 0 \\
\hline $12 / a b r$ & $17: 45$ & $18: 10$ & 25 & $25 / a b r$ & 0 & 0 & 0 & 0 & 0 \\
\hline $13 / \mathrm{abr}$ & $15: 04$ & $15: 25$ & 21 & $28 / a b r$ & 0 & 0 & 0 & 25 & \\
\hline $13 / \mathrm{abr}$ & $15: 04$ & $15: 25$ & 21 & $30 / \mathrm{abr}$ & 0 & 0 & 0 & 0 & 0 \\
\hline
\end{tabular}

Fonte: (PEREIRA, 2016).

A tabela acima serviu como base para gerar os gráficos de controle a seguir, sendo a Figura 5 o Gráfico das médias e a Figura 6 o Gráfico das amplitudes.
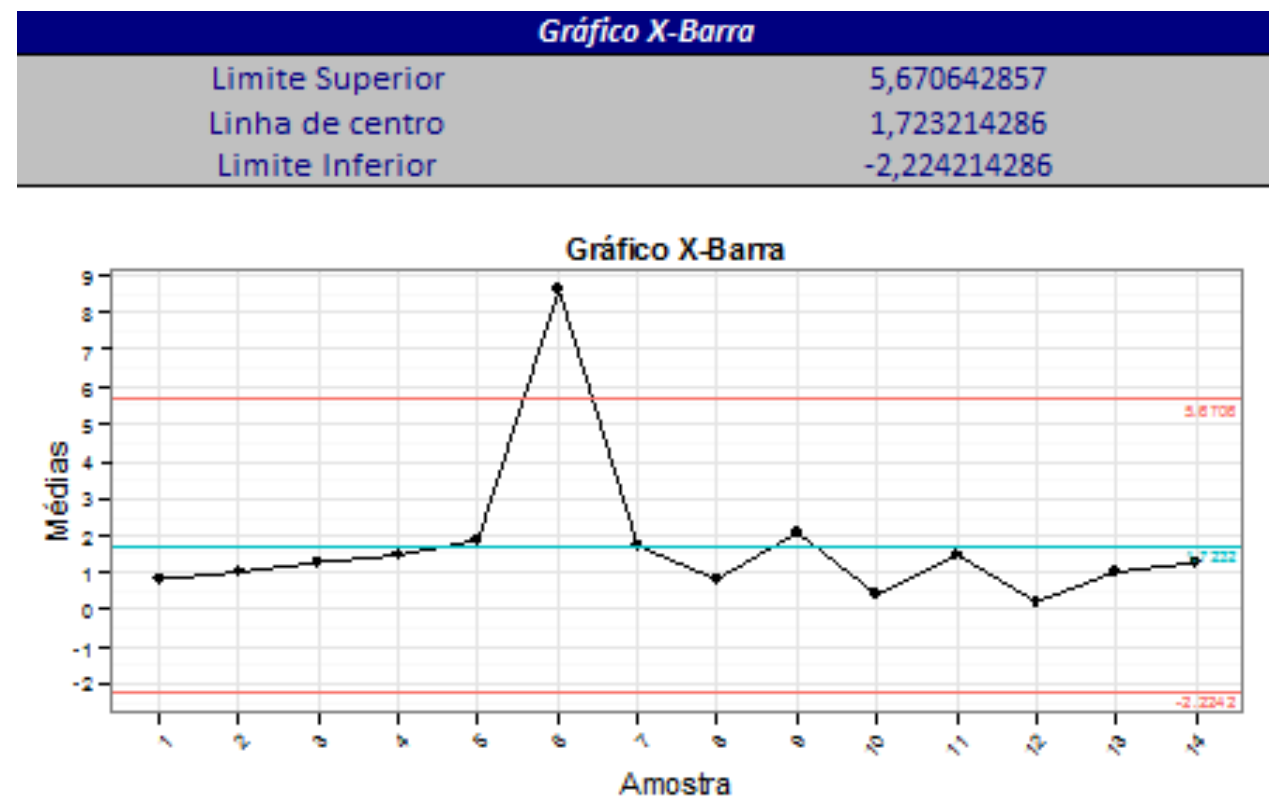

Figura 05. Análise de Variáveis do CEP: Gráfico das médias.

Fonte: (PEREIRA, 2016). 


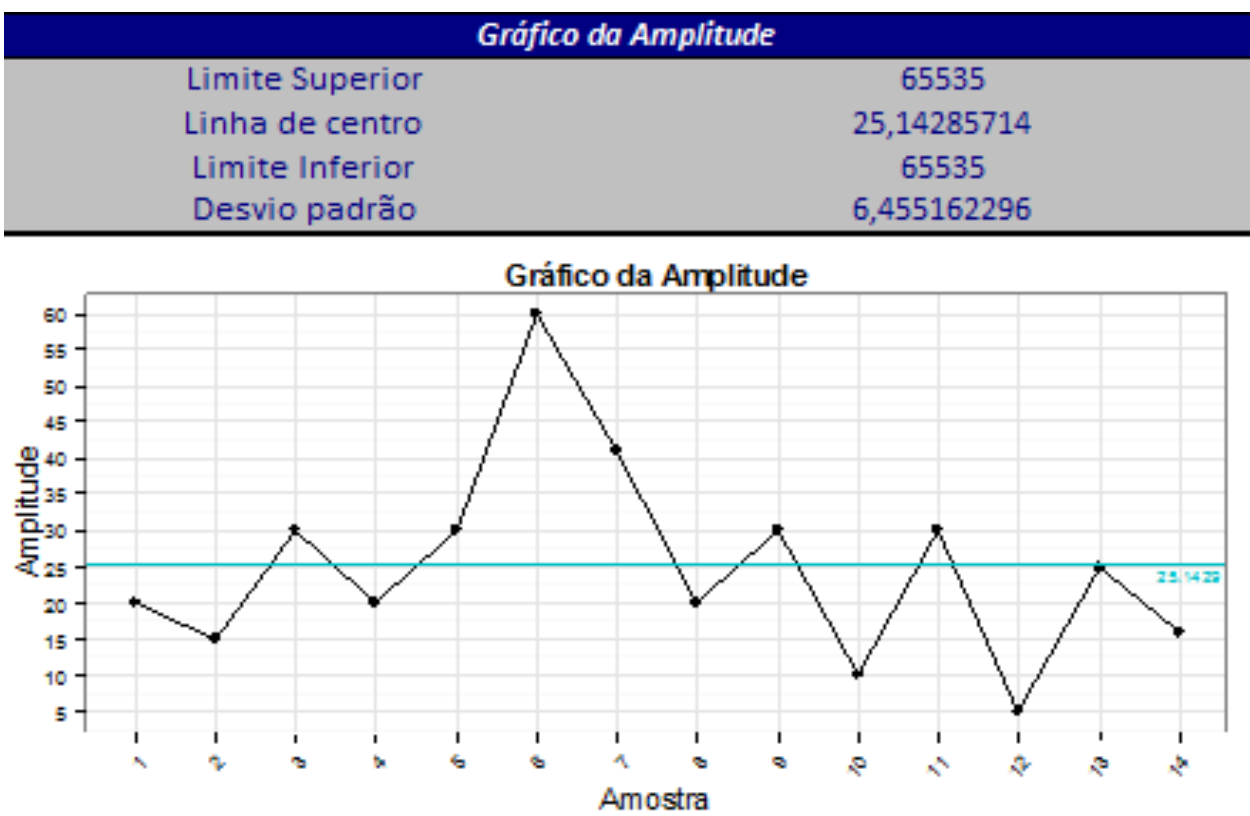

Figura 06. Análise de Variáveis do CEP: Gráfico das amplitudes.

Fonte: (PEREIRA, 2016).

É possível ver que há uma amostra que ultrapassa o limite de controle, essa parada aconteceu no dia 12 , é necessário uma analise mais criteriosa dessa parada para realizar um plano de ação que erradique a causa da mesma.

\section{CONCLUSÃO}

Após o estudo das ferramentas da qualidade, e sua aplicação na linha de envase da indústria de alimentos e bebidas, foi possível descobrir as falhas principais do processo, e averiguar quais deveriam ser priorizadas no momento. A partir da exposição dos resultados ficou clara a contribuição significativa das mesmas, tanto para a constatação desses eventuais problemas, quanto para tomadas de decisões que podem levar a empresa a excelência.
Com o auxilio do Diagrama de Pareto identificamos no processo produtivo a maior responsável pelas paradas existentes, apresentando a Tampadeira uma frequência acumulada de 32\%. O Diagrama de Ishikawa nos indicou as causas e seus efeitos referente a essa parada, levando em consideração mão de obra, materiais, máquinas, métodos, meio ambiente e medição. Com o Diagrama de Dispersão analisamos se havia correlação entre a média de eficiência do frasco e seu fornecedor, com a quantidade de caixas produzidas e obtivemos uma relação de $69,61 \%$. Já a Carta Controle determinou se o processo produtivo estava operando dentro do padrão estatístico, ou seja, se as paradas frequentes estavam dentro do limite superior e inferior, e visualizamos que apenas uma amostra ultrapassou o limite de controle. 
Portanto orientou-se a realizar e seguir um plano de ação por meio das causas principais sinalizadas no Diagrama de Ishikawa, adquirir um espaço maior para instalar a linha e diminuir o congestionamento, aumentar as esteiras da linha de produção, orientar os funcionários, desenvolver auditoria em fornecedores, aplicar treinamentos de reciclagem para criar uma mentalidade responsável nos colaboradores, atribuindo a todos 0 comprometimento com a qualidade em cada etapa de produção, e aplicar treinamentos específicos com os operadores, para que eles consigam realizar a regulagem sem agredir os equipamentos e tenham a consciência da importância em anotar os dados corretamente.

\section{REFERÊNCIAS}

BARROS, E.; BONIFANI, F. Ferramentas da qualidade. São Paulo: Pearson Edcation do Brasil, 2014.

CUSTÓDIO, M. F. Gestão da qualidade e produtividade. São Paulo: Pearson Education do Brasil, 2015.

SELEME, R.; HUMBERTO, S. Controle de qualidade: as ferramentas essenciais. Curitiba: InterSaberes, 2012. 